\title{
Experiences of out-of-hours task-shifting from GPs: a systematic review of qualitative studies
}

\author{
Emily Lyness ${ }^{1 *}$, Jennifer Parker ${ }^{1}$, Merlin L Willcox ${ }^{1}$, Hajira Dambha-Miller ${ }^{1}$ \\ ${ }^{1}$ Primary Care Research Centre, Faculty of Medicine, University of Southampton, \\ Aldermoor Health Centre, Aldermoor Close, Southampton, UK
}

\begin{abstract}
Background: The current GP workforce is insufficient to manage rising demand in patient care within out-of-hours $(\mathrm{OOH})$ primary care services. To meet this challenge, non-medical practitioners (NMPs) are employed to fulfil tasks traditionally carried out by GPs. It is important to learn from experiences of task-shifting in this setting to inform optimal delivery of care.
\end{abstract}

Aim: To synthesise qualitative evidence of experiences of task-shifting in the $\mathrm{OOH}$ primary care setting.

Design \& setting: Systematic review of qualitative studies and thematic synthesis.

Method: Electronic searches were conducted across CINAHL (Cumulative Index of Nursing and Allied Health Literature), PsychINFO, Cochrane, MEDLINE, Embase, and OpenGrey for qualitative studies of urgent or $\mathrm{OOH}$ primary care services, utilising task-shifting or role delegation. Included articles were quality appraised and key findings collated through thematic synthesis.

Results: A total of 2497 studies were screened, of which six met the inclusion criteria. These included interviews with 15 advanced nurse practitioners (ANPs), three physician assistants (PAs), two paramedics, and a focus group of 22 GPs, and focus groups with 33 nurses. Key findings highlight the importance of clearly defining and communicating the scope of practice of NMPs, and of building their confidence by appropriate training, support, and mentoring.

Conclusion: While NMPs may have the potential to make a substantial contribution to $\mathrm{OOH}$ primary care services, there has been very little research on experiences of task-shifting. Evidence to date highlights the need for further training specific to $\mathrm{OOH}$ services. Mentorship and support to manage

*For correspondence: emilylyness@doctors.org.uk

Competing interest: See page 8

Received: 08 March 2021

Accepted: 19 April 2021

Published: 21 July 2021

(C)This article is Open Access: CC BY license (https://creativecommons.org/licenses/by/4.0/)

Author Keywords: primary health care, after-hours care, task-shifting, workforce, out of hours

Copyright (C) 2021, The Authors; DOI:10.3399/BJGPO.2021.0043 the sometimes challenging cases presenting to $\mathrm{OOH}$ could enable more effective $\mathrm{OOH}$ services and better patient care.

\section{How this fits in}

Demand for OOH GP services is increasing, but the existing GP workforce is insufficient to meet this need. Government policy proposes a greater skill mix of NMPs in the workforce to shift tasks away from GPs. Early studies suggest that NMPs deliver safe and effective care; however, there is limited evidence specific to the $\mathrm{OOH}$ setting. In some settings, NMPs have a defined scope of practice, which excludes certain patient groups. If this is clearly communicated, GPs will focus on more complex patients, which NMPs cannot see. This may increase GP workload within shifts but the involvement of NMPs reduces the number of shifts that GPs need to work. If the NMPs' scope of practice is not clearly defined, communicated, or recognised, the service will run less efficiently, giving rise to negative team perceptions and poor interprofessional relationships. Building confidence of NMPs 
to work autonomously takes dedicated training, support, and mentoring, and is critical to efficient running of $\mathrm{OOH}$ services.

\section{Introduction}

$\mathrm{OOH}$ primary care services deliver 'urgent care' outside normal working hours. NHS England defines urgent care as 'the range of responses that healthcare services provide to people who require urgent advice and treatment'. ${ }^{1,2}$ Demand for $\mathrm{OOH}$ services is increasing in the UK: in 2014, they received 8.6 million calls and completed 6.8 million assessments; 2.9 million via telephone, 0.9 million home visits, and 3 million face-to-face consultations, contributing to a cost of $£ 400$ million. ${ }^{3}$ Historically it has been challenging to establish data for comparison owing to variation in recording, changing definitions of $\mathrm{OOH}$, and different methodologies of collecting this data. ${ }^{4}$ However, it is expected that demand will continue to rise owing to increasing levels of multimorbidity, complex health needs, and changes in service utilisation behaviour. ${ }^{5-7}$

The existing GP workforce is insufficient to meet $\mathrm{OOH}$ demand. In response, the government has proposed the development of a greater skill mix of NMPs. NMPs include advanced practitioners who are employed to undertake roles traditionally filled by GPs and can, therefore, shift tasks away from GPs. Although it is likely that task-shifting is not a novel practice, it is difficult to ascertain the current picture of task-shifting in $\mathrm{OOH}$ care in England owing to the wide variety in NMP roles. A 2017 study found over 500 different job titles for advance practice roles in health care and care pathway organisation. $^{8}$

The skill mix of NMPs working in $\mathrm{OOH}$ has historically comprised advanced practitioners from a variety of clinical backgrounds, including primary and emergency services. The effectiveness of task-shifting from GPs to NMPs is still debated, particularly concerning managing complexity. Earlier studies suggest that NMPs are able to deliver safe and effective care with positive patient-reported outcomes, high satisfaction, and decreased costs. However, there is limited $\mathrm{OOH}$ specific evidence. ${ }^{9-11}$ One UK trial compared in-hours management of same-day requested assessments, including home visits, between GPs and ANPs and found equivalent outcomes. ${ }^{10}$ A Dutch quasi-experimental study examining task-shifting from GPs to nurses in $\mathrm{OOH}$ found similar consultation numbers, but a higher medical complexity in patients treated by GPs. ${ }^{12}$ Along with the acutely unwell and children aged $<5$ years, there is evidence that more potentially complex patient groups, such as adults aged $>65$ years and those with chronic diseases from socioeconomically deprived areas, are accessing care in the evenings. ${ }^{6}$ While this may partly be owing to barriers to daytime access, there is also a growing culture of 24-hour service demand contributing to $\mathrm{OOH}$ service utilisation behaviour. ${ }^{5,6,13}$

Furthermore, because of the variety of practitioners in $\mathrm{OOH}$, there is a lack of clarity around NMP role definition and competency expectations. ${ }^{14,15}$ This uncertainty has led to negative team perceptions and poor interprofessional relationships with less effective service delivery. ${ }^{16-19}$ In turn, there are challenges recruiting NMPs to $\mathrm{OOH}$ settings. ${ }^{19-24}$

This study aimed to understand the experiences of $\mathrm{OOH}$ staff in addressing these issues, to find out how task-shifting can be implemented to optimise service effectiveness and patient care. Therefore, a systematic review was conducted of qualitative studies on experiences of task-shifting to NMPs from GPs in $\mathrm{OOH}$ primary care services.

\section{Method}

ENTREQ guidelines ${ }^{25}$ were followed and the study was registered with PROSPERO.

\section{Search strategy and inclusion and exclusion criteria}

A pre-planned systematic literature search was performed (database inception to October 2020) across CINAHL, PsychINFO, Cochrane, MEDLINE, Embase, and OpenGrey. No restrictions on publication date, country, or language were used. In addition to the electronic database search, references of included studies were manually searched for relevant articles. The detailed search strategy is provided in Supplementary Table S1. Experts in eight countries (Austria, Denmark, Germany, the Netherlands, Norway, Sweden, UK, and US) were also contacted in April 2021 to ask whether they knew of any relevant studies. Inclusion criteria were empirical studies with qualitative methods or 
mixed methods with a qualitative component reporting on task-shifting from GPs to NMPs (including nurses, paramedics, and PAs) within the $\mathrm{OOH}$ primary care setting (outside of routine opening hours).

\section{Study selection and data extraction}

All titles and abstracts were initially screened by a single author, then two authors independently screened a $10 \%$ sample. ${ }^{26}$ Full-text versions of potentially eligible articles were reviewed in duplicate and any ambiguity resolved by team discussion (see Supplementary Figure S1). Data were extracted from the results sections of included articles using NVivo (version 1.3) in preparation for thematic synthesis.

\section{Results synthesis}

Thematic synthesis was undertaken as outlined by Thomas and Harden, ${ }^{27}$ including coding of text line by line followed by the development of descriptive themes, and then generation of analytical themes. Each article was initially read and relevant qualitative data highlighted to produce a conceptual framework. This allowed iterative formation of codes relating to experiences of $\mathrm{OOH}$ task-shifting. Line-by-line examination of the data allowed inductive exploration and identification of recurring patterns describing individual experiences and perspectives. Common themes were identified in team discussion, which built on the framework. Findings were then summarised using a narrative approach and were drafted into a summary by one author, then commented on by the other authors. Through several rounds of discussions, an iterative process of hierarchical ordering of themes was repeated until analytical themes describing and/or explaining initial descriptive themes were present, and team consensus reached. This ordering of themes enabled a deeper understanding of the unifying concepts to explain experiences of task-shifting in $\mathrm{OOH}$.

\section{Risk of bias}

The Critical Appraisal Skills Programme (CASP) assessment tool for qualitative research ${ }^{28,29}$ was used by two researchers to independently appraise the studies; disagreements were resolved by discussion. This assessment was not used to judge weight of findings, or exclude studies, but to highlight potential bias in the results of included studies.

\section{Results}

\section{Study selection}

The search identified 2497 articles (see Supplementary Figure S1). After removal of duplicates and screening, 42 articles were selected for full-text screening. Team discussion excluded a further 36 articles because they were not about $\mathrm{OOH}$ activities such as home visits or consultations. Six articles were included in the review. Study characteristics are summarised in Supplementary Table S2. Four were small UK-based, mixed-methods studies with qualitative components, published between 2011 and 2019; two reported experiences of ANPs conducting home visits in Bristol and Cumbria; ${ }^{11,19}$ one reported a pilot of integrating US-trained PAs into an $\mathrm{OOH}$ service in Scotland; ${ }^{30}$ and one reported the evaluation of a programme to train paramedics to work in $\mathrm{OOH}$ in Bristol. ${ }^{31}$ One study in Norway investigated the experiences of nurses triaging patients presenting with respiratory symptoms, ${ }^{32}$ and one study in the Netherlands explored the views of GPs and nurse practitioners (NPs) taking part in a quasi-experimental study into optimal skill mix in $\mathrm{OOH}$ primary care. ${ }^{33}$

\section{Quality of included studies}

The CASP quality assessment (see Supplementary Table S3) identified weaknesses in the reporting of recruitment strategy by three studies. ${ }^{19,30,31}$ Farmer et al did not specify how many PAs were included in focus groups nor how they were recruited, but an appropriately broad sample was described. ${ }^{30}$ Farmer et al also omitted reporting of the interview method, data management, and analysis approach, which limits appraisal of whether this data addressed the research question. ${ }^{30}$ Moule et al provided a limited description of the thematic approach used to analyse the responses of the three interviews carried out. ${ }^{31}$ Yuill did not state the qualitative analysis technique employed. ${ }^{19}$ The reflexivity of the research team was not reported by four studies. ${ }^{19,30,31,33}$ Ethical issues were not fully explored by Lindberg et al. ${ }^{32}$ 


\section{Summary of themes}

Two main factors were identified that influenced both job satisfaction and efficiency of NMPs in $\mathrm{OOH}$ services: 1) a clearly defined skillset, scope of practice, and role; and 2) confidence (see Supplementary figure S2). These could be enhanced by clear communication of the roles, recognition and collaborative working by GPs, and by training, support, and mentorship from GPs. Additional illustrative quotes are available in Supplementary Table S4.

\section{Theme 1: Clearly defined skillset, scope of practice, and role of NMPs Defining scope of practice and role}

Some $\mathrm{OOH}$ services appeared to expect NMPs to function as GPs, although they do not have the same breadth and depth of knowledge and skills. Therefore, it is not surprising that they felt unprepared for certain patients and sometimes avoided them if possible:

'We tend to see them all as equal, but they do have different experiences and abilities.' (GPs talking about ANP home visiting) ${ }^{11}$

[ANPs] mentioned that certain groups of patients are more complex than others and their experience had not always prepared them for this. Common areas included palliative, children's and mental health care, providing telephone advice, managing comorbidities and wider prescribing ... [they] would avoid specific patient groups, including children and people with mental health issues, but they would see such patients if they were the only clinician on the shift. ${ }^{19}$

Rather than expecting NMPs to see all patients, the GP cooperative studied in the Netherlands specifically defined certain patient groups as outside the scope of practice of NMPs: patients aged $<1$ year, patients suffering psychiatric complaints, abdominal pain, chest pain, a neck ailment, headache, or dizziness. ${ }^{33}$ This enabled the service to run efficiently as long as GPs accepted and collaborated by focusing their work on patients that NMPs could not see:

'If there are two NPs we have to check all patients on the presentation list, not only their urgency level, but also their complaint. If it's, for example, an ear infection, I take the next patient and leave the ear infection to the NP.' (GP in $\mathrm{OOH}$ cooperative) $)^{33}$

Van der Biezen et al described a clear trade-off in terms of the impact on GP workload of employing more ANPs. When ANPs worked more shifts, GPs' workload was reduced in terms of the number of shifts they were required to work; however, during shifts with more ANPs, GPs' workload was increased in terms of longer consultations with more complex cases. ${ }^{33}$ On the other hand, NMPs brought some unique skills, which in some cases enabled them to work more efficiently than GPs:

'NPs ask less often for support from the medical assistant compared to GPs; for example, regarding putting on bandages after suturing. ${ }^{33}$

\section{Recognition of NMPs' skillset and role}

It is important that colleagues recognise the unique skills offered by NMPs and their role. Greater appreciation of different NMP training backgrounds could avoid potential misunderstandings that occur when roles are viewed as equivalent. In some cases, GPs did not understand the scope of practice of ANPs. ANPs described needing to gain the trust of colleagues in primary and secondary care, who were uncertain about the NMPs' roles. They had some negative experiences of patient contact, especially if clinical triage had first been carried out by a GP:

'A lack of understanding about the ANP title and competencies, by patients and other members of the public as well as colleagues including GPs, nurses and other primary and secondary care staff ... this is exacerbated by the wide variety of people calling themselves advanced practitioners or nurse practitioners who do not necessarily have the requisite experience or qualifications to undertake the role. ${ }^{19}$

Greater clarity of role expectation enabled NMPs to feel valued and satisfied in their jobs, and clearer communication was needed to ensure the whole team was informed: 
'PAs reported working most effectively and were most satisfied where there was a distinct gap in a team that they could fill; for example, in the $\mathrm{OOH}$ clinic they worked like a supporting GP to a lead GP. ${ }^{30}$

'At the start of the shift, I say, "I don't treat those patients" ... then you start doing consultations. Making agreements with the GP who starts later isn't really working, you're either busy yourself, or the door of the GP is already closed.' (NP in OOH cooperative) ${ }^{33}$

Understanding the scope of practice and limitations of NMPs was emphasised as critical to avoid inappropriate task allocation and to ensure the service runs efficiently:

'Some shifts run perfectly, others you think "I wish there was a third GP now." Especially when there are GPs who pick out patients with skin complaints from the presentation list. You get delay if more patients with abdominal pain show up.' (Medical assistant in $\mathrm{OOH}$ cooperative) ${ }^{33}$

\section{Theme 2: NMP confidence}

\section{NMP confidence is critical for an efficient service}

Lack of confidence was commonly reported by NMPs, leading to anxiety and inefficiencies in the service. Nurses conducting telephone triage reported offering GP appointments to some patients who didn't need them, because they were worried about missing serious illness, and lacked the confidence to reassure patients and their parents over the phone. ${ }^{32}$ This led to both slower triage (as the nurses felt the need to document the history in detail) and excessive numbers of GP appointments:

'You never sit in that chair and feel confident.' (ANP doing telephone triage) ${ }^{32}$

Lack of confidence was also given as a reason for NMPs working more slowly in face-to-face consultations:

'I must say a patient with, for example, a sore, I finish those consultations within a minute and I feel confident to do that. I think NPs are more careful and still take a full history just to be sure they do their work properly.' (GP in $\mathrm{OOH}$ cooperative) ${ }^{33}$

Some nurses also described lacking confidence in making referrals to secondary care:

'For nurses, having the confidence to talk to doctors about complex presentations and diagnoses can be very challenging, but once you get used to it, it becomes easier ... "19

Inadequate preparation could exacerbate challenges faced by NMPs and contribute to a feeling of isolation when starting roles in $\mathrm{OOH}$ teams:

'Daytime [practice has] more protection and barriers ... the step into $\mathrm{OOH}$ was a big one ... unknown ... no exposure to what is being seen [with] more acute unwell patients.' (ANP home visiting) ${ }^{11}$

\section{Training, clinical support, and mentorship help to build confidence and} autonomy

Individualised training and support were suggested as key for NMPs who may be less experienced in traditional GP tasks, particularly at the beginning of a career. However, it was recognised that the need decreased with time as NMPs gained experience. Investing in role-specific training, particularly at induction, could minimise potential role uncertainty and help NMPs feel valued as part of the professional team:

'Working autonomously in home visits, ANPs reported: "when you start in an organisation, you don't know who to go to ... as you learn, you know who you can go to for support." There was consensus among the interviewees that having some protected time for learning on- and offshift would be beneficial ... "makes them feel valued and that we do care about their continuing professional development."19 
NMPs viewed the clinical training required to undertake their roles as an opportunity for career development, while maintaining clinical practice. Practitioners who are trained to specified professional standards are able to take more clinical responsibilities, which facilitates task-shifting:

'It was felt that providing training on a sliding scale would be useful for different healthcare practitioners. A nurse experienced in independent practice in another environment may only need 8-weeks training to achieve the competencies; however, paramedics may need 16 weeks to achieve the competencies. ${ }^{131}$

Professional dialogue, case discussion, and peer learning within the $\mathrm{OOH}$ team could minimise professional hierarchy, especially within nurse-doctor relationships:

'Peer support was mentioned as positive ... participants talked about learning from clinical colleagues, sharing experiences ... one[ANP] mentioned that: "Nurses always think hierarchically, that GPs are better than you is the thought/perception, but when you are learning together ... you realise that they don't know things, so you are like them, which breaks down the barriers." "19

Clinical mentorship was flagged as an effective way to support NMPs in a different way to clinical training. This should be regular, consistent, and accessible, particularly in the first year of practice, but also throughout their professional career development:

'ANPs acknowledged that the knowledge and support of the GPs was important, although with time this was only required occasionally.' (ANP home visiting) ${ }^{11}$

'GPs and ANPs suggested: "a structured ANP home-visiting induction ... needs to be developed to include typical home-visit scenarios, shadow shifts, and a mentorship programme ... the opportunity for clinical supervision, case-review discussion, ongoing audit of cases, and a forum to debrief."'11

Specific support from a designated GP was considered beneficial in ensuring NMP confidence. This may promote job satisfaction and reduce anxiety for those who lack confidence even to approach a GP to ask for support:

[Paramedic] students recommended concentrated time spent with one allocated mentor and that GP support is increased. ${ }^{31}$

NMPs wanted to practice autonomously and found this to be an attractive aspect of their role; however, it could also provoke stress. Therefore, having access to GP support was still necessary to provide reassurance, validation of clinical decisions, and to build confidence and autonomous practice:

[Anxiety] about working alone, knowing that support, often by phone, was available but not always immediately contributed to a feeling of isolation and stress as they experienced the need for autonomous decision making ... "19

'Some [ANPs] said one of the most enjoyable aspects was being able to make decisions "knowing your "own sphere of competence", treating patients within this and referring on as appropriate were important."

\section{Discussion}

\section{Summary}

There have been very few qualitative studies on experiences of task-shifting from GPs to NMPs in the $\mathrm{OOH}$ primary care setting. This study highlights the importance of clearly defining NMPs' roles and scope of practice. In addition, the importance of providing training, support, and mentoring is shown in order to develop their confidence to practice autonomously and efficiently.

\section{Strengths and limitations}

A comprehensive electronic search of the literature was conducted, complemented by grey literature, manual searching, and contacting experts. Although 2497 papers were screened, only six matched the inclusion criteria, from only three European countries. The system of $\mathrm{OOH}$ primary care varies 
in different countries so it is possible that task-shifting activities to NMPs have long existed in many countries and is, therefore, not novel, resulting in minimal clinical research published in this area. ${ }^{34}$

The low numbers of participants included in the studies raises the possibility of recruitment bias and limits transferability of these findings, even within the UK. Additionally, not all qualitative studies included were of high quality, particularly those that were part of a mixed-methods approach. However, this reflects the available literature and emphasises the need for more research around NMPs and $\mathrm{OOH}$ task-shifting.

The review team included GPs with experience of $\mathrm{OOH}$ work. While this provides strength to the analytical approach and contextual understanding of the subject, it may have affected interpretation of findings through the authors' assumptions made based on their own experiences; however, the authors attempted to guard against this by strictly limiting themselves to what was reported in the included studies.

\section{Comparison with existing literature}

NMPs can deliver safe and effective in-hours primary care, positive patient-reported outcomes, and decreased organisational costs, but there is limited data on long-term outcomes for medical complexity and multimorbidity. ${ }^{14,22-24,35}$ The findings of the present study suggest appropriate training, mentorship, and interprofessional collaboration can enable NMPs to feel supported in the management of a wider variety of cases in $\mathrm{OOH}$, which promotes job satisfaction, professional development, and efficiency of the service. These findings are consistent with the recognition that NMPs, specifically nurses, desire collaboration to mitigate potential imbalances in workload and dissatisfaction that can occur when working in a subsidiary role. The concept of complexity itself is a continuum dependent on the clinical presentation, and there is evidence that ANPs may be more likely to manage those socially complex cases ${ }^{36}$ while GPs focus on medically complex patients. Thus, a collaborative approach is likely to be useful and relevant to the $\mathrm{OOH}$ population.

Much of the evidence for NMP task-shifting comes from non-urgent care such as nurse-led chronic care. ${ }^{20,35,37-39}$ The Royal College of General Practitioners and Health Education England have designed a Core Capabilities Framework for advanced practice in primary care. ${ }^{40}$ This brings into alignment nursing professionals in primary care. Although not specific to $\mathrm{OOH}$, there is overlap. This framework could reduce uncertainty about NMP role definition and scope of practice.

However, for those NMPs already in the $\mathrm{OOH}$ workforce, there remains some confusion about professional identity and respect for clinical autonomy. Professional identity not only encompasses clinical autonomy, but also confidence and aspiration in professional development. The literature examining role transfer to advanced practitioners supports the importance of mentorship and supervision. This is likely to be vital in encouraging retention of NMPs and encouraging professional development into leadership roles, thus contributing to the sustainability of the $\mathrm{OOH}$ workforce. ${ }^{20,23,39,41}$

\section{Implications for research and practice}

NMPs have the potential to support growing $\mathrm{OOH}$ workloads through task-shifting. Additional support must include context-specific role preparation, clinical mentoring, and a collaborative approach to sharing the caseload efficiently. Standardised core competency frameworks incorporated into current training would engender uniformity and trust in the advanced practitioner role in $\mathrm{OOH}$ primary care, and would be relevant to other healthcare settings where NMPs share tasks. However, more research is needed to better understand the optimal team composition, taking into account the trade-off between decreasing the number of GP shifts and increasing the workload for GPs during shifts when more NMPs are working. Where scope of practice has been defined by some $\mathrm{OOH}$ services, this is not consistent and appears somewhat arbitrary. Better evidence is needed to underpin decisions and definitions regarding scope of practice for NMPs in $\mathrm{OOH}$ services. Further qualitative research is needed into the experiences of NMPs (especially paramedics and PAs) in a wider range of $\mathrm{OOH}$ services in different countries. It is important to improve understanding of the barriers and enablers to service efficiency and NMP job satisfaction in a range of roles, including not only home visits, but also face-to-face and telephone triage consultations.

While NMPs have the potential to make a substantial contribution to the $\mathrm{OOH}$ setting, understanding the experiences of those involved in task-shifting and addressing their specific professional training needs is vital to prepare NMPs for the $\mathrm{OOH}$ setting. Clear definition and communication of roles, 
coupled with mentorship and support, could improve efficiency of $\mathrm{OOH}$ services and patient care. More evidence is needed to fully understand the experiences of $\mathrm{OOH}$ staff undertaking task-shifting in $\mathrm{OOH}$ primary care and how this can be improved.

\section{Funding}

Emily Lyness is a National Institute for Health Research (NIHR) Academic Clinical Fellow. Hajira Dambha-Miller is a NIHR funded Academic Clinical Lecturer. Merlin L Willcox is an academic GP and his salary was funded by NIHR (grant reference number: CL-2016-26-005). The views and opinions expressed by authors in this publication are those of the authors and do not necessarily reflect those of the NIHR or the Department of Health and Social Care.

\section{Ethical approval}

Ethical approval was not required for this review. An exemption was obtained from the University of Southampton Ethics Committee (Ethics and Research Governance Online reference number: 58095).

Trial registration number

PROSPERO (reference number: CRD42020218866)

\section{Provenance}

Freely submitted; externally peer reviewed.

\section{Competing interests}

Hajira Dambha-Miller is the Editor-in-Chief of BJGP Open, but had no involvement in the peer review process or decision on this manuscript.

\section{References}

1. NHS England. Integrated urgent care service specification. 2017; https://www.england.nhs.uk/wp-content/uploads/ 2014/06/Integrated-Urgent-Care-Service-Specification.pdf (accessed 30 Jun 2021).

2. NHS England. Next steps on the NHS five year forward view. 2017; https://www.england.nhs.uk/wp-content/ uploads/2017/03/NEXT-STEPS-ON-THE-NHS-FIVE-YEAR-FORWARD-VIEW.pdf (accessed 30 Jun 2021).

3. National Audit Office. Out-of-hours GP services in England. 2014; https://www.nao.org.uk/wp-content/uploads/ 2014/09/Out-of-hours-GP-services-in-England1.pdf (accessed 9 Jul 2021).

4. Salisbury C, Trivella M, Bruster S. Demand for and supply of out of hours care from general practitioners in England and Scotland: observational study based on routinely collected data. BMJ 2000; 320(7235): 618-621. DOI: https:// doi.org/10.1136/bmj.320.7235.618

5. Kelly SJ, Piercy H, lbbotson R, Fowler Davis SV. Who attends out-of-hours general practice appointments? Analysis of a patient cohort accessing new out-of-hours units. BMJ Open 2018; 8(6): e020308. DOI: https://doi.org/10. 1136/bmjopen-2017-020308

6. Foster $\mathrm{H}$, Moffat KR, Burns $\mathrm{N}$, et al. What do we know about demand, use and outcomes in primary care out-ofhours services? A systematic scoping review of international literature. BMJ Open 2020; 10(1): e033481. DOI: https://doi.org/10.1136/bmjopen-2019-033481

7. Hobbs FDR, Bankhead C, Mukhtar T, et al. Clinical workload in UK primary care: a retrospective analysis of 100 million consultations in England, 2007-14. Lancet 2016; 387(10035): 2323-2330. DOI: https://doi.org/10.1016/ S0140-6736(16)00620-6

8. Leary A, Maclaine K, Trevatt $P$, et al. Variation in job titles within the nursing workforce. J Clin Nurs 2017; 26(2324): 4945-4950. DOI: https://doi.org/10.1111/jocn.13985

9. Smits $M$, Peters $Y$, Ranke $S$, et al. Substitution of general practitioners by nurse practitioners in out-of-hours primary care home visits: a quasi-experimental study. Int J Nurs Stud 2020; 104: 103445. DOI: https://doi.org/10. 1016/j.ijnurstu.2019.103445

10. Edwards M, Bobb C, Robinson SI. Nurse practitioner management of acute in-hours home visit or assessment requests: a pilot study. Br J Gen Pract 2009; 59(558): 7-11. DOI: https://doi.org/10.3399/bjgp09X394798

11. Collins D. Assessing the effectiveness of advanced nurse practitioners undertaking home visits in an out of hours urgent primary care service in England. J Nurs Manag 2019; 27(2): 450-458. DOI: https://doi.org/10.1111/jonm. 12680

12. Smits $\mathrm{M}$, Peters $\mathrm{Y}$, Ranke $\mathrm{S}$, et al. Substitution of general practitioners by nurse practitioners in out-of-hours primary care home visits: a quasi-experimental study. Int J Nurs Stud 2020; 104: 103445. DOI: https://doi.org/10. 1016/j.ijnurstu.2019.103445

13. Keizer $E$, Smits $M$, Peters $Y$, et al. Contacts with out-of-hours primary care for nonurgent problems: patients' beliefs or deficiencies in healthcare? BMC Fam Pract 2015; 16: 157. DOI: https://doi.org/10.1186/s12875-015-0376-9

14. Hooker RS, Moloney-Johns AJ, McFarland MM. Patient satisfaction with physician assistant/associate care: an international scoping review. Hum Resour Health 2019; 17(1): 104. DOI: https://doi.org/10.1186/s12960-019-0428- 
15. Tavares W, Boet S. On the assessment of paramedic competence: a narrative review with practice implications. Prehosp Disaster Med 2016; 31(1): 64-73. DOI: https://doi.org/10.1017/S1049023X15005166

16. Torrens $C$, Campbell $P$, Hoskins $G$, et al. Barriers and facilitators to the implementation of the advanced nurse practitioner role in primary care settings: a scoping review. Int J Nurs Stud 2020; 104: 103443. DOI: https://doi.org/ 10.1016/j.jinurstu.2019.103443

17. Memmel J, Spalsbury M. Urgent care medicine and the role of the APP within this specialty. Dis Mon 2017; 63(5): 105-114. DOI: https://doi.org/10.1016/j.disamonth.2017.03.001

18. Imison C, Castle-Clarke S, Watson R. Reshaping the workforce to deliver the care patients need. London: Nuffield Trust; 2016.

19. Yuill J. The role and experiences of advanced nurse practitioners working in out of hours urgent care services in a primary care setting. Nurs Manage 2018; 25(2): 18-23. DOI: https://doi.org/10.7748/nm.2018.e1745

20. Evans C, Poku B, Pearce R, et al. Characterising the evidence base for advanced clinical practice in the UK: a scoping review protocol. BMJ Open 2020; 10(5): e036192. DOI: https://doi.org/10.1136/bmjopen-2019-036192

21. Thompson J, McNall A, Tiplady S, et al. Whole systems approach. J Health Organ Manag 2019; 33(4): 443-459. DOI: https://doi.org/10.1108/JHOM-11-2018-0337

22. Schofield B, Voss S, Proctor A, et al. Exploring how paramedics are deployed in general practice and the perceived benefits and drawbacks: a mixed-methods scoping study. BJGP Open 2020; 4(2): bjgpopen20X101037. DOI: https://doi.org/10.3399/bjgpopen20X101037

23. Elliott N. Building leadership capacity in advanced nurse practitioners - the role of organisational management. J Nurs Manag 2017; 25(1): 77-81. DOI: https://doi.org/10.1111/jonm.12444

24. Anderson $\mathrm{H}$, Birks $\mathrm{Y}$, Adamson J. Exploring the relationship between nursing identity and advanced nursing practice: an ethnographic study. J Clin Nurs 2020; 29(7-8): 1195-1208. DOI: https://doi.org/10.1111/jocn.15155

25. Tong $A$, Flemming $K$, Mclnnes $E$, et al. Enhancing transparency in reporting the synthesis of qualitative research: ENTREQ. BMC Med Res Methodol 2012; 12: 181. DOI: https://doi.org/10.1186/1471-2288-12-181

26. Ouzzani M, Hammady H, Fedorowicz Z, Elmagarmid A. Rayyan - a web and mobile APP for systematic reviews. Syst Rev 2016; 5(1): 210. DOI: https://doi.org/10.1186/s13643-016-0384-4

27. Thomas J, Harden A. Methods for the thematic synthesis of qualitative research in systematic reviews. BMC Med Res Methodol 2008; 8: 45. DOI: https://doi.org/10.1186/1471-2288-8-45

28. Williams V, Boylan A-M, Nunan D. Critical appraisal of qualitative research: necessity, partialities and the issue of bias. BMJ Evid Based Med 2020; 25(1): 9-11. DOI: https://doi.org/10.1136/bmjebm-2018-111132

29. Critical Appraisal Skills Programme. CASP checklist: 10 questions to help you make sense of a systematic review. 2018; https://casp-uk.net/wp-content/uploads/2018/01/CASP-Systematic-Review-Checklist_2018.pdf (accessed 18 Jun 2021).

30. Farmer J, Currie M, Hyman J, et al. Evaluation of physician assistants in National Health Service Scotland. Scott Med J 2011; 56(3): 130-134. DOI: https://doi.org/10.1258/smj.2011.011109

31. Moule $P$, Clompus $S$, Lockyer $L$, et al. Preparing non-medical clinicians to deliver GP out-of-hours services: lessons learned from an innovative approach. Educ Prim Care 2018; 29(6): 376-380. DOI: https://doi.org/10.1080/ 14739879.2018.1516517

32. Lindberg $\mathrm{BH}$, Rebnord IK, Høye S. Phone triage nurses' assessment of respiratory tract infections - the tightrope walk between gatekeeping and service providing. A qualitative study. Scand J Prim Health Care 2021: 1-9. DOI: https://doi.org/10.1080/02813432.2021.1908715

33. van der Biezen $M$, Wensing $M$, Poghosyan $L$, et al. Collaboration in teams with nurse practitioners and general practitioners during out-of-hours and implications for patient care; a qualitative study. BMC Health Serv Res 2017; 17(1): 589. DOI: https://doi.org/10.1186/s12913-017-2548-x

34. Berchet $\mathrm{C}$, Nader $\mathrm{C}$. The organisation of out-of-hours primary care in OECD countries. OECD Health Working Papers, No 89. Paris: OECD Publishing; 2016. DOI: https://doi.org//10.1787/5jlr3czbqw23-en

35. Martínez-González NA, Djalali S, Tandjung R, et al. Substitution of physicians by nurses in primary care: a systematic review and meta-analysis. BMC Health Serv Res 2014; 14: 214. DOI: https://doi.org/10.1186/1472-6963-14-214

36. Cody R, Gysin S, Merlo C, et al. Complexity as a factor for task allocation among general practitioners and nurse practitioners: a narrative review. BMC Fam Pract 2020; 21(1): 38. DOI: https://doi.org/10.1186/s12875-020-1089-2

37. Martin-Misener R, Harbman $P$, Donald $F$, et al. Cost-effectiveness of nurse practitioners in primary and specialised ambulatory care: systematic review. BMJ Open 2015; 5(6): e007167. DOI: https://doi.org/10.1136/bmjopen-2014007167

38. Ashley C, Halcomb E, Brown A. Transitioning from acute to primary health care nursing: an integrative review of the literature. J Clin Nurs 2016; 25(15-16): 2114-2125. DOI: https://doi.org/10.1111/jocn.13185

39. Evans $C$, Pearce R, Greaves $S$, Blake H. Advanced clinical practitioners in primary care in the UK: a qualitative study of workforce transformation. Int J Environ Res Public Health 2020; 17(12): 4500. DOI: https://doi.org/10. 3390/ijerph17124500

40. Royal College of General Practitioners. Core Capabilities Framework for Advanced Clinical Practice (Nurses) Working in General Practice / Primary Care in England. 2020; https://www.hee.nhs.uk/sites/default/files/ documents/ACP\%20Primary\%20Care\%20Nurse\%20Fwk\%202020.pdf (accessed 9 Jul 2021).

41. Bryant-Lukosius $D$, Spichiger $E$, Martin J, et al. Framework for evaluating the impact of advanced practice nursing roles. J Nurs Scholarsh 2016; 48(2): 201-209. DOI: https://doi.org/10.1111/jnu.12199 